Bull. Korean Math. Soc. 52 (2015), No. 2, pp. 603-618

http://dx.doi.org/10.4134/BKMS.2015.52.2.603

\title{
SOME SYMMETRY IDENTITIES FOR GENERALIZED TWISTED BERNOULLI POLYNOMIALS TWISTED BY UNRAMIFIED ROOTS OF UNITY
}

\author{
DAE SAN Kim
}

\begin{abstract}
We derive three identities of symmetry in two variables and eight in three variables related to generalized twisted Bernoulli polynomials and generalized twisted power sums, both of which are twisted by unramified roots of unity. The case of ramified roots of unity was treated previously. The derivations of identities are based on the $p$-adic integral expression, with respect to a measure introduced by Koblitz, of the generating function for the generalized twisted Bernoulli polynomials and the quotient of $p$-adic integrals that can be expressed as the exponential generating function for the generalized twisted power sums.
\end{abstract}

\section{Introduction and preliminaries}

Let $p$ be a fixed prime. Throughout this paper, $\mathbb{Z}_{p}, \mathbb{Q}_{p}, \mathbb{C}_{p}$ will respectively denote the ring of $p$-adic integers, the field of $p$-adic rational numbers and the completion of the algebraic closure of $\mathbb{Q}_{p}$. Assume that $|\cdot|_{p}$ is the normalized absolute value of $\mathbb{C}_{p}$, such that $|p|_{p}=\frac{1}{p}$. The group $\Gamma$ of all roots of unity of $\mathbb{C}_{p}$ is the direct product of its subgroups $\Gamma_{u}$ (the subgroup of unramified roots of unity) and $\Gamma_{r}$ (the subgroup of ramified roots of unity). Namely,

$$
\Gamma=\Gamma_{u} \cdot \Gamma_{r}, \quad \Gamma_{u} \cap \Gamma_{r}=\{1\},
$$

where

$$
\begin{aligned}
\Gamma_{u} & =\left\{\xi \in \mathbb{C}_{p} \mid \xi^{r}=1 \text { for some } r \in \mathbb{Z}_{>0} \text { with }(r, p)=1\right\}, \\
\Gamma_{r} & =\left\{\xi \in \mathbb{C}_{p} \mid \xi^{p^{s}}=1 \text { for some } s \in \mathbb{Z}_{>0}\right\} .
\end{aligned}
$$

Let $d$ be a fixed positive integer. Then we let

$$
X=X_{d}=\lim _{\overleftarrow{N}} \mathbb{Z} / d p^{N} \mathbb{Z}=\cup_{a=0}^{d p-1}\left(a+d p \mathbb{Z}_{p}\right),
$$

Received April 23, 2014; Revised August 23, 2014.

2010 Mathematics Subject Classification. 11B68, 11S80, 05A19.

Key words and phrases. generalized twisted Bernoulli polynomial, generalized twisted power sum, Dirichlet character, unramified roots of unity, $p$-adic integral, identities of symmetry. 
with

$$
a+d p^{N} \mathbb{Z}_{p}=\left\{x \in X \mid x \equiv a\left(\bmod d p^{N}\right)\right\},
$$

and let $\pi: X \rightarrow \mathbb{Z}_{p}$ be the map given by the inverse limit of the natural maps

$$
\mathbb{Z} / d p^{N} \mathbb{Z} \rightarrow \mathbb{Z} / p^{N} \mathbb{Z} \text {. }
$$

If $g$ is a function on $\mathbb{Z}_{p}$, we will use the same notation to denote the function $g \circ \pi$. Let $\chi:(\mathbb{Z} / d \mathbb{Z})^{*} \rightarrow \overline{\mathbb{Q}}^{*}$ be a (primitive) Dirichlet character of conductor $d$. Then it will be pulled back to $X$ via the natural map $X \rightarrow \mathbb{Z} / d \mathbb{Z}$. Here we fix, once and for all, an imbedding $\overline{\mathbb{Q}} \rightarrow \mathbb{C}_{p}$, so that $\chi$ is regarded as a map of $X$ to $\mathbb{C}_{p}$.

Let $z \in \mathbb{C}_{p}$ be such that $z^{d p^{N}} \neq 1$ for all $N$. Then we define

$$
\mu_{z}\left(a+d p^{N} \mathbb{Z}_{p}\right)=\frac{z^{a}}{z^{d p^{N}}-1} .
$$

Observe that (1) is -1 times of the corresponding one in [18]. Then it is known (cf. [18]) that $\mu_{z}$ extends to a measure on $X$ if and only if $z \in\{x \in$ $\left.\mathbb{C}_{p}|| x-\left.1\right|_{p} \geq 1\right\}$. So for any such a $z$, and any continuous $\mathbb{C}_{p}$-valued function $f$ on $X$,

$$
\begin{aligned}
\int_{X} f(x) d \mu_{z}(x) & =\lim _{N \rightarrow \infty} \sum_{a=0}^{d p^{N}-1} f(a) \mu\left(a+d p^{N} \mathbb{Z}_{p}\right) \\
& =\lim _{N \rightarrow \infty} \frac{1}{z^{d p^{N}}-1} \sum_{a=0}^{d p^{N}-1} f(a) z^{a} .
\end{aligned}
$$

Throughout this paper, we let $\xi \neq 1$ be any fixed $r$-th root of 1 , with $(r, p d)=1$ (and hence $\xi \in \Gamma_{u}$ ), and let

$$
E=\left\{\left.t \in \mathbb{C}_{p}|| t\right|_{p}<p^{-\frac{1}{p-1}}\right\} .
$$

Then $u_{\xi}$ is a measure on $X$, and, for a positive integer $w, \mu_{\xi w}$ is a measure provided that $w$ is not divisible by $r$. For each fixed $t \in E$ the function $e^{z t}$ is analytic on $\mathbb{Z}_{p}$ and hence considered as a function on $X$. Using the definition (2), we get the $p$-adic integral expression of the generating function for the generalized twisted Bernoulli numbers $B_{n, \chi, \xi}$ attached to $\chi$ and $\xi$;

$$
\begin{aligned}
t \int_{X} \chi(z) e^{z t} d \mu_{\xi}(z) & =\frac{t}{\xi^{d} e^{d t}-1} \sum_{a=0}^{d-1} \chi(a) \xi^{a} e^{a t} \\
& =\sum_{n=0}^{\infty} B_{n, \chi, \xi} \frac{t^{n}}{n !} \quad(t \in E) .
\end{aligned}
$$

So we have the following $p$-adic integral expression of the generating function for the generalized twisted Bernoulli polynomials $B_{n, \chi, \xi}(x)$ attached to $\chi$ and 
$\xi$

$$
\begin{aligned}
t \int_{X} \chi(z) e^{(x+z) t} d \mu_{\xi}(z) & =\frac{t e^{x t}}{\xi^{d} e^{d t}-1} \sum_{a=0}^{d-1} \chi(a) \xi^{a} e^{a t} \\
& =\sum_{n=0}^{\infty} B_{n, \chi, \xi}(x) \frac{t^{n}}{n !} \quad\left(t \in E, x \in \mathbb{Z}_{p}\right) .
\end{aligned}
$$

Also, from (2) we have the $p$-adic integral expression of the generating function for the twisted Bernoulli numbers $B_{n, \xi}$ :

$$
\begin{aligned}
t \int_{X} e^{z t} d \mu_{\xi}(z) & =\frac{t}{\xi e^{t}-1} \\
& =\sum_{n=0}^{\infty} B_{n, \xi} \frac{t^{n}}{n !} \quad(t \in E) .
\end{aligned}
$$

Thus we obtain the $p$-adic integral expression of the generating function for the twisted Bernoulli polynomials $B_{n, \xi}(x)$ :

$$
t \int_{X} e^{(x+z) t} d \mu_{\xi}(z)=\frac{t e^{x t}}{\xi e^{t}-1}=\sum_{n=0}^{\infty} B_{n, \xi}(x) \frac{t^{n}}{n !} \quad\left(t \in E, x \in \mathbb{Z}_{p}\right) .
$$

Let $S_{k}(n ; \chi, \xi)$ denote the $k$-th generalized twisted power sum of the first $n+1$ nonnegative integers attached to $\chi$ and $\xi$, namely

$$
S_{k}(n ; \chi, \xi)=\sum_{a=0}^{n} \chi(a) \xi^{a} a^{k}=\chi(0) \xi^{0} 0^{k}+\chi(1) \xi^{1} 1^{k}+\cdots+\chi(n) \xi^{n} n^{k} .
$$

From (4), (6), and (7), one easily derives the following identities: for $w \in$ $\mathbb{Z}_{>0}$, with $w$ not divisible by $r$,

$$
\begin{aligned}
\frac{\int_{X} \chi(x) e^{x t} d \mu_{\xi}(x)}{\int_{X} e^{d w y t} d \mu_{\xi d w}(y)} & =\frac{\xi^{d w} e^{d w t}-1}{\xi^{d} e^{d t}-1} \sum_{a=0}^{d-1} \chi(a) \xi^{a} e^{a t} \\
& =\sum_{a=0}^{d w-1} \chi(a) \xi^{a} e^{a t} \\
& =\sum_{k=0}^{\infty} S_{k}(d w-1 ; \chi, \xi) \frac{t^{k}}{k !} \quad(t \in E)
\end{aligned}
$$

In what follows, we will always assume that the $p$-adic integrals of the various (twisted) exponential functions on $X$ are defined for $t \in E$ (cf. (3)), and therefore it will not be mentioned.

One may refer to [1], [2], [3], [11], [12], [14], [15], [16], [17], [19], [20], and [21] for some of the previous works on identities of symmetry in two variables involving Bernoulli polynomials and power sums, and for some related results. For the brief history, one is referred to those papers. For the first time, the idea of [11] was extended in [10] to the case of three variables so as to yield 
many new identities with abundant symmetry. This added some new identities of symmetry even to the existing ones in two variables as well. Also, see [4], $[5],[7],[8]$, and [9] for some other extensions of the idea of [11] to the case of three variables.

On the other hand, in [13] the author obtained identities of symmetry in two variables involving generalized twisted Bernoulli polynomials and generalized twisted power sums, both of which are twisted by ramified roots of unity (i.e., $p$-power roots of unity). In [6], this was also extended to the case of three variables. In these ramified cases, $p$-adic Volkenborn-type integrals are used in both [6] and [13].

In this paper, in order to treat the unramified roots of unity case (i.e., the orders of the roots of unity are prime to $p$ and the conductors of Dirichlet characters), we will adopt the measure introduced by Koblitz (cf. [18]) instead of Volkenborn measure, as stated in (1) and (2). It seems this idea has never been exploited before. In the end, we will be able to derive three identities of symmetry in two variables (cf. (44)-(46)) and eight in three variables related to generalized twisted Bernoulli polynomials and generalized twisted power sums (cf. (47)-(50), (53)-(56)).

The following is stated as Theorem 8 and an example of the full six symmetries in any positive integers $w_{1}, w_{2}, w_{3}$, with $w_{1} w_{2} w_{3}$ not divisible by $r$.

$$
\begin{aligned}
& w_{1}^{n} \sum_{k=0}^{n}\left(\begin{array}{l}
n \\
k
\end{array}\right) \sum_{a=0}^{d w_{1}-1} \chi(a) \xi^{a w_{2} w_{3}} B_{k, \chi, \xi^{a w_{1} w_{3}}}\left(w_{2} y_{1}+\frac{w_{2}}{w_{1}} a\right) S_{n-k}\left(d w_{3}-1 ; \chi, \xi^{w_{1} w_{2}}\right) w_{2}^{n-k} w_{3}^{k} \\
& =w_{1}^{n} \sum_{k=0}^{n}\left(\begin{array}{l}
n \\
k
\end{array}\right) \sum_{a=0}^{d w_{1}-1} \chi(a) \xi^{a w_{2} w_{3}} B_{k, \chi, \xi^{w_{1} w_{2}}}\left(w_{3} y_{1}+\frac{w_{3}}{w_{1}} a\right) S_{n-k}\left(d w_{2}-1 ; \chi, \xi^{w_{1} w_{3}}\right) w_{3}^{n-k} w_{2}^{k} \\
& =w_{2}^{n} \sum_{k=0}^{n}\left(\begin{array}{l}
n \\
k
\end{array}\right) \sum_{a=0}^{d w_{2}-1} \chi(a) \xi^{a w_{1} w_{3}} B_{k, \chi, \xi^{w_{2} w_{3}}}\left(w_{1} y_{1}+\frac{w_{1}}{w_{2}} a\right) S_{n-k}\left(d w_{3}-1 ; \chi, \xi^{w_{1} w_{2}}\right) w_{1}^{n-k} w_{3}^{k} \\
& =w_{2}^{n} \sum_{k=0}^{n}\left(\begin{array}{l}
n \\
k
\end{array}\right) \sum_{a=0}^{d w_{2}-1} \chi(a) \xi^{a w_{1} w_{3}} B_{k, \chi, \xi^{w_{1} w_{2}}}\left(w_{3} y_{1}+\frac{w_{3}}{w_{2}} a\right) S_{n-k}\left(d w_{1}-1 ; \chi, \xi^{w_{2} w_{3}}\right) w_{3}^{n-k} w_{1}^{k} \\
& =w_{3}^{n} \sum_{k=0}^{n}\left(\begin{array}{l}
n \\
k
\end{array}\right) \sum_{a=0}^{d w_{3}-1} \chi(a) \xi^{a w_{1} w_{2}} B_{k, \chi, \xi^{w_{2} w_{3}}}\left(w_{1} y_{1}+\frac{w_{1}}{w_{3}} a\right) S_{n-k}\left(d w_{2}-1 ; \chi, \xi^{w_{1} w_{3}}\right) w_{1}^{n-k} w_{2}^{k} \\
& =w_{3}^{n} \sum_{k=0}^{n}\left(\begin{array}{l}
n \\
k
\end{array}\right) \sum_{a=0}^{d w_{3}-1} \chi(a) \xi^{a w_{1} w_{2}} B_{k, \chi, \xi_{1} w_{3}}\left(w_{2} y_{1}+\frac{w_{2}}{w_{3}} a\right) S_{n-k}\left(d w_{1}-1 ; \chi, \xi^{w_{2} w_{3}}\right) w_{2}^{n-k} w_{1}^{k} .
\end{aligned}
$$

The derivations of identities are based on the $p$-adic integral expression of the generating function for the generalized twisted Bernoulli polynomials in (5) and the quotient of integrals in (8)-(10) that can be expressed as the exponential generating function for the generalized twisted power sums. These abundance of symmetries would not be unearthed if such $p$-adic integral representations had not been available. We indebted this idea to the paper [11]. 


\section{Several types of quotients of $p$-adic integrals in two variables}

Here we will introduce several types of quotients of $p$-adic integrals on $X$ or $X^{2}$ from which some interesting identities follow owing to the built-in symmetries in $w_{1}, w_{2}$. In the following, $w_{1}, w_{2}$ are positive integers with suitable restrictions and the explicit expression of integral in (11) is obtained from the identities in (4) and (6).

( $\alpha$ ) Type $\Gamma^{i}($ for $i=0,1,2)$

$I\left(\Gamma^{i}\right)=\frac{\int_{X^{2}} \chi\left(x_{1}\right) \chi\left(x_{2}\right) t^{2-i} e^{\left(w_{1} x_{1}+w_{2} x_{2}+w_{1} w_{2}\left(\sum_{j=1}^{2-i} y_{j}\right)\right) t} d \mu_{\xi^{w_{1}}}\left(x_{1}\right) d \mu_{\xi^{w_{2}}}\left(x_{2}\right)}{\left(\int_{X} e^{d w_{1} w_{2} x_{3} t} d \mu_{\xi^{d w_{1} w_{2}}}\left(x_{3}\right)\right)^{i}}$

$$
=\frac{t^{2-i} e^{w_{1} w_{2}\left(\sum_{j=1}^{2-i} y_{j}\right) t}\left(\xi^{d w_{1} w_{2}} e^{d w_{1} w_{2} t}-1\right)^{i}\left(\sum_{a=0}^{d-1} \chi(a) \xi^{a w_{1}} e^{a w_{1} t}\right)\left(\sum_{a=0}^{d-1} \chi(a) \xi^{a w_{2}} e^{a w_{2} t}\right)}{\left(\xi^{d w_{1}} e^{d w_{1} t}-1\right)\left(\xi^{d w_{2}} e^{d w_{2} t}-1\right)} .
$$

The above $p$-adic integral is invariant under the transposition of $w_{1}, w_{2}$ as one can see either from the $p$-adic integral representation in (11) or from its explicit evaluation in (12).

\section{Several types of quotients of $p$-adic integrals in three variables}

Here we will introduce several types of quotients of $p$-adic integrals on $X$ or $X^{3}$ from which some interesting identities follow due to the built-in symmetries in $w_{1}, w_{2}, w_{3}$. In the following, $w_{1}, w_{2}, w_{3}$ are positive integers with suitable restrictions and all of the explicit expressions of integrals in (14), (16), (18), and (20) are obtained from the identities in (4) and (6).

(a) Type $\Lambda_{23}^{i}$ (for $\left.i=0,1,2,3\right)$

$$
\begin{aligned}
& \int_{X^{3}} \chi\left(x_{1}\right) \chi\left(x_{2}\right) \chi\left(x_{3}\right) t^{3-i} e^{\left(w_{2} w_{3} x_{1}+w_{1} w_{3} x_{2}+w_{1} w_{2} x_{3}+w_{1} w_{2} w_{3}\left(\sum_{j=1}^{3-i} y_{j}\right)\right) t}
\end{aligned}
$$

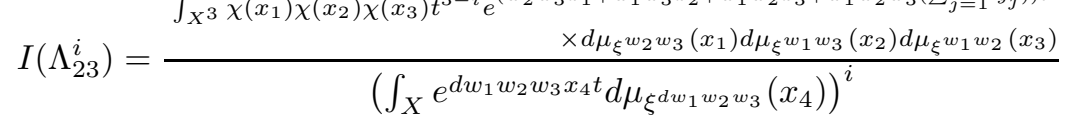

$$
\begin{aligned}
& =\frac{t^{3-i} e^{w_{1} w_{2} w_{3}\left(\sum_{j=1}^{3-i} y_{j}\right) t}\left(\xi^{d w_{1} w_{2} w_{3}} e^{d w_{1} w_{2} w_{3} t}-1\right)^{i}}{\left(\xi^{d w_{2} w_{3}} e^{d w_{2} w_{3} t}-1\right)\left(\xi^{d w_{1} w_{3}} e^{d w_{1} w_{3} t}-1\right)\left(\xi^{d w_{1} w_{2}} e^{d w_{1} w_{2} t}-1\right)} \\
& \times\left(\sum_{a=0}^{d-1} \chi(a) \xi^{a w_{2} w_{3}} e^{a w_{2} w_{3} t}\right)\left(\sum_{a=0}^{d-1} \chi(a) \xi^{a w_{1} w_{3}} e^{a w_{1} w_{3} t}\right)\left(\sum_{a=0}^{d-1} \chi(a) \xi^{a w_{1} w_{2}} e^{a w_{1} w_{2} t}\right) .
\end{aligned}
$$

Here $w_{2} w_{3}, w_{1} w_{3}, w_{1} w_{2}$ are not divisible by $r$, for $i=0$, and $w_{1} w_{2} w_{3}$ is not divisible by $r$, for $i=1,2,3$.

(b) Type $\Lambda_{13}^{i}($ for $i=0,1,2,3)$

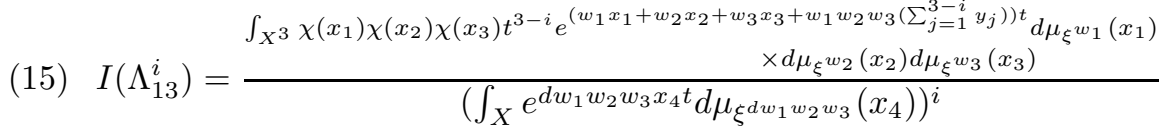




$$
\begin{aligned}
= & \frac{t^{3-i} e^{w_{1} w_{2} w_{3}\left(\sum_{j=1}^{3-i} y_{j}\right) t}\left(\xi^{d w_{1} w_{2} w_{3}} e^{d w_{1} w_{2} w_{3} t}-1\right)^{i}}{\left(\xi^{d w_{1}} e^{d w_{1} t}-1\right)\left(\xi^{d w_{2}} e^{d w_{2} t}-1\right)\left(\xi^{d w_{3}} e^{d w_{3} t}-1\right)} \\
& \left.\times\left(\sum_{a=0}^{d-1} \chi(a) \xi^{a w_{1}} e^{a w_{1} t}\right)\left(\sum_{a=0}^{d-1} \chi(a) \xi^{a w_{2}} e^{a w_{2} t}\right)\left(\sum_{a=0}^{d-1} \chi(a) \xi^{a w_{3}} e^{a w_{3} t}\right)\right) .
\end{aligned}
$$

Here $w_{1}, w_{2}, w_{3}$ are not divisible by $r$, for $i=0$, and $w_{1} w_{2} w_{3}$ is not divisible by $r$, for $i=1,2,3$.

(c-0) Type $\Lambda_{12}^{0}$

$I\left(\Lambda_{12}^{0}\right)=\int_{X^{3}} \chi\left(x_{1}\right) \chi\left(x_{2}\right) \chi\left(x_{3}\right) t^{3} e^{\left(w_{1} x_{1}+w_{2} x_{2}+w_{3} x_{3}+w_{2} w_{3} y+w_{1} w_{3} y+w_{1} w_{2} y\right) t}$

$$
\begin{aligned}
& \text { (17) } \times d \mu_{\xi^{w_{1}}}\left(x_{1}\right) d \mu_{\xi^{w_{2}}}\left(x_{2}\right) d \mu_{\xi^{w_{3}}}\left(x_{3}\right) \\
& \text { (18) }=\frac{t^{3} e^{\left(w_{2} w_{3}+w_{1} w_{3}+w_{1} w_{2}\right) y t}\left(\sum_{a=0}^{d-1} \chi(a) \xi^{a w_{1}} e^{a w_{1} t}\right)\left(\sum_{a=0}^{d-1} \chi(a) \xi^{a w_{2}} e^{a w_{2} t}\right)\left(\sum_{a=0}^{d-1} \chi(a) \xi^{a w_{3}} e^{a w_{3} t}\right)}{\left(\xi^{d w_{1}} e^{d w_{1} t}-1\right)\left(\xi^{d w_{2}} e^{d w_{2} t}-1\right)\left(\xi^{d w_{3}} e^{d w_{3} t}-1\right)} .
\end{aligned}
$$

Here $w_{1}, w_{2}, w_{3}$ are not divisible by $r$.

(c-1) Type $\Lambda_{12}^{1}$

$$
\begin{aligned}
& I\left(\Lambda_{12}^{1}\right)=\frac{\int_{X^{3}} \chi\left(x_{1}\right) \chi\left(x_{2}\right) \chi\left(x_{3}\right) e^{\left(w_{1} x_{1}+w_{2} x_{2}+w_{3} x_{3}\right) t} d \mu_{\xi^{w_{1}}}\left(x_{1}\right) d \mu_{\xi^{w_{2}}}\left(x_{2}\right) d \mu_{\xi^{w_{3}}}\left(x_{3}\right)}{\int_{X} e^{d w_{2} w_{3} z_{1} t} d \mu_{\xi^{d w_{2} w_{3}}}\left(z_{1}\right) \int_{X} e^{d w_{1} w_{3} z_{2} t} d \mu_{\xi^{d w_{1} w_{3}}}\left(z_{2}\right) \int_{X} e^{d w_{1} w_{2} z_{3} t} d \mu_{\xi^{d w_{1} w_{2}}}\left(z_{3}\right)} \\
& =\frac{\left(\xi^{d w_{2} w_{3}} e^{d w_{2} w_{3} t}-1\right)\left(\xi^{d w_{1} w_{3}} e^{d w_{1} w_{3} t}-1\right)\left(\xi^{d w_{1} w_{2}} e^{d w_{1} w_{2} t}-1\right)}{\left(\xi^{d w_{1}} e^{d w_{1} t}-1\right)\left(\xi^{d w_{2}} e^{d w_{2} t}-1\right)\left(\xi^{d w_{3}} e^{d w_{3} t}-1\right)} \\
& \times\left(\sum_{a=0}^{d-1} \chi(a) \xi^{a w_{1}} e^{a w_{1} t}\right)\left(\sum_{a=0}^{d-1} \chi(a) \xi^{a w_{2}} e^{a w_{2} t}\right)\left(\sum_{a=0}^{d-1} \chi(a) \xi^{a w_{3}} e^{a w_{3} t}\right) .
\end{aligned}
$$

Here $w_{2} w_{3}, w_{1} w_{3}, w_{1} w_{2}$ are not divisible by $r$.

All of the above $p$-adic integrals of various types are invariant under all permutations of $w_{1}, w_{2}, w_{3}$, as one can see either from $p$-adic integral representations in (13), (15), (17), and (19) or their explicit evaluations in (14), (16), (18), and (20).

\section{Identities for generalized twisted Bernoulli polynomials in two variables}

All of the following results can be easily obtained from (5) and (8)-(10). $(\alpha-0)$

$$
\begin{aligned}
I\left(\Gamma^{0}\right) & =\int_{X} \chi\left(x_{1}\right) t e^{w_{1}\left(x_{1}+w_{2} y_{1}\right) t} d \mu_{\xi^{w_{1}}}\left(x_{1}\right) \int_{X} \chi\left(x_{2}\right) t e^{w_{2}\left(x_{2}+w_{1} y_{2}\right) t} d \mu_{\xi^{w_{2}}}\left(x_{2}\right) \\
& =\left(\sum_{k=0}^{\infty} B_{k, \chi, \xi^{w_{1}}}\left(w_{2} y_{1}\right) \frac{\left(w_{1} t\right)^{k}}{k !}\right)\left(\sum_{l=0}^{\infty} B_{l, \chi, \xi^{w_{2}}}\left(w_{1} y_{2}\right) \frac{\left(w_{2} t\right)^{l}}{l !}\right) \\
21) & =\sum_{n=0}^{\infty}\left(\sum_{k=0}^{n}\left(\begin{array}{l}
n \\
k
\end{array}\right) B_{k, \chi, \xi^{w_{1}}}\left(w_{2} y_{1}\right) B_{n-k, \chi, \xi^{w_{2}}}\left(w_{1} y_{2}\right) w_{1}^{k} w_{2}^{n-k}\right) \frac{t^{n}}{n !} .
\end{aligned}
$$

$(\alpha-1)$ Here we write $I\left(\Gamma^{1}\right)$ in two different ways: 
$(22)$

$$
\begin{aligned}
& I\left(\Gamma^{1}\right)=\int_{X} \chi\left(x_{1}\right) t e^{w_{1}\left(x_{1}+w_{2} y_{1}\right) t} d \mu_{\xi^{w_{1}}}\left(x_{1}\right) \times \frac{\int_{X} \chi\left(x_{2}\right) e^{w_{2} x_{2} t} d \mu_{\xi^{w_{2}}}\left(x_{2}\right)}{\int_{X} e^{d w_{1} w_{2} x_{3} t} d \mu_{\xi^{d w_{1} w_{2}}}\left(x_{3}\right)} \\
& =\left(\sum_{k=0}^{\infty} B_{k, \chi, \xi^{w_{1}}}\left(w_{2} y_{1}\right) \frac{\left(w_{1} t\right)^{k}}{k !}\right)\left(\sum_{l=0}^{\infty} S_{l}\left(d w_{1}-1 ; \chi, \xi^{w_{2}}\right) \frac{\left(w_{2} t\right)^{l}}{l !}\right) \\
& =\sum_{n=0}^{\infty}\left(\sum_{k=0}^{n}\left(\begin{array}{l}
n \\
k
\end{array}\right) B_{k, \chi, \xi^{w_{1}}}\left(w_{2} y_{1}\right) S_{n-k}\left(d w_{1}-1 ; \chi, \xi^{w_{2}}\right) w_{1}^{k} w_{2}^{n-k}\right) \frac{t^{n}}{n !} .
\end{aligned}
$$

(2) Invoking (9), (22) can also be written as

$$
\begin{aligned}
& I\left(\Gamma^{1}\right)=\sum_{a=0}^{d w_{1}-1} \chi(a) \xi^{a w_{2}} \int_{X} \chi\left(x_{1}\right) t e^{w_{1}\left(x_{1}+w_{2} y_{1}+\frac{w_{2}}{w_{1}} a\right) t} d \mu_{\xi^{w_{1}}}\left(x_{1}\right) \\
& =\sum_{a=0}^{d w_{1}-1} \chi(a) \xi^{a w_{2}}\left(\sum_{n=0}^{\infty} B_{n, \chi, \xi^{w_{1}}}\left(w_{2} y_{1}+\frac{w_{2}}{w_{1}} a\right) \frac{\left(w_{1} t\right)^{n}}{n !}\right) \\
& =\sum_{n=0}^{\infty}\left(w_{1}^{n} \sum_{a=0}^{d w_{1}-1} \chi(a) \xi^{a w_{2}} B_{n, \chi, \xi^{w_{1}}}\left(w_{2} y_{1}+\frac{w_{2}}{w_{1}} a\right)\right) \frac{t^{n}}{n !} .
\end{aligned}
$$

$(\alpha-2)$

$$
\begin{aligned}
& I\left(\Gamma^{2}\right)=\frac{\int_{X} \chi\left(x_{1}\right) e^{w_{1} x_{1} t} d \mu_{\xi^{w_{1}}}\left(x_{1}\right)}{\int_{X} e^{d w_{1} w_{2} x_{3} t} d \mu_{\xi^{d w_{1} w_{2}}}\left(x_{3}\right)} \times \frac{\int_{X} \chi\left(x_{2}\right) e^{w_{2} x_{2} t} d \mu_{\xi^{w_{2}}}\left(x_{2}\right)}{\int_{X} e^{d w_{1} w_{2} x_{3} t} d \mu_{\xi^{d w_{1} w_{2}}}\left(x_{3}\right)} \\
& =\left(\sum_{k=0}^{\infty} S_{k}\left(d w_{2}-1 ; \chi, \xi^{w_{1}}\right) \frac{\left(w_{1} t\right)^{k}}{k !}\right)\left(\sum_{l=0}^{\infty} S_{l}\left(d w_{1}-1 ; \chi, \xi^{w_{2}}\right) \frac{\left(w_{2} t\right)^{l}}{l !}\right) \\
& =\sum_{n=0}^{\infty}\left(\sum_{k=0}^{n}\left(\begin{array}{l}
n \\
k
\end{array}\right) S_{k}\left(d w_{2}-1 ; \chi, \xi^{w_{1}}\right) S_{n-k}\left(d w_{1}-1 ; \chi, \xi^{w_{2}}\right) w_{1}^{k} w_{2}^{n-k}\right) \frac{t^{n}}{n !} .
\end{aligned}
$$

\section{Identities for generalized twisted Bernoulli polynomials in three}

\section{variables}

All of the following results can be easily obtained from (5) and (8)-(10). First, let's consider Type $\Lambda_{23}^{i}$, for each $i=0,1,2,3$.

$(\mathrm{a}-0)$

$$
\begin{aligned}
& I\left(\Lambda_{23}^{0}\right)=\int_{X} \chi\left(x_{1}\right) t e^{w_{2} w_{3}\left(x_{1}+w_{1} y_{1}\right) t} d \mu_{\xi^{w_{2} w_{3}}}\left(x_{1}\right) \int_{X} \chi\left(x_{2}\right) t e^{w_{1} w_{3}\left(x_{2}+w_{2} y_{2}\right) t} d \mu_{\xi_{1}^{w_{1} w_{3}}\left(x_{2}\right)} \\
& \times \int_{X} \chi\left(x_{3}\right) t e^{w_{1} w_{2}\left(x_{3}+w_{3} y_{3}\right) t} d \mu_{\xi^{w_{1} w_{2}}}\left(x_{3}\right)
\end{aligned}
$$

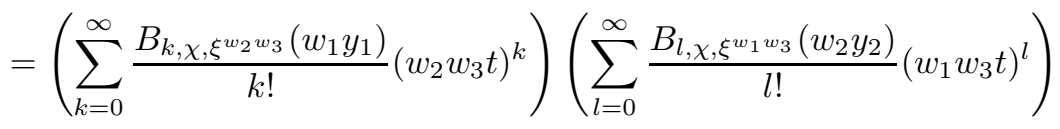




$$
\begin{aligned}
& \times\left(\sum_{m=0}^{\infty} \frac{B_{m, \chi, \xi^{w} w_{2}}\left(w_{3} y_{3}\right)}{m !}\left(w_{1} w_{2} t\right)^{m}\right)
\end{aligned}
$$

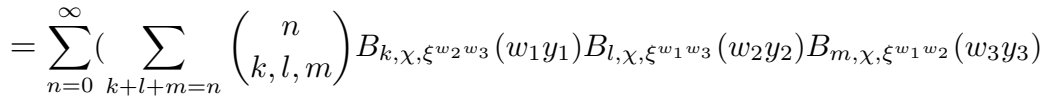

$$
\begin{aligned}
& \left.\times w_{1}^{l+m} w_{2}^{k+m} w_{3}^{k+l}\right) \frac{t^{n}}{n !},
\end{aligned}
$$

where the inner sum is over all nonnegative integers $k, l, m$, with $k+l+m=n$ and

$$
\left(\begin{array}{c}
n \\
k, l, m
\end{array}\right)=\frac{n !}{k ! l ! m !} .
$$

(a-1) Here we write $I\left(\Lambda_{23}^{1}\right)$ in two different ways:

$$
\begin{aligned}
& I\left(\Lambda_{23}^{1}\right)=\int_{X} \chi\left(x_{1}\right) t e^{w_{2} w_{3}\left(x_{1}+w_{1} y_{1}\right) t} d \mu_{\xi^{w_{2} w_{3}}}\left(x_{1}\right) \int_{X} \chi\left(x_{2}\right) t e^{w_{1} w_{3}\left(x_{2}+w_{2} y_{2}\right) t} d \mu_{\xi^{w_{1} w_{3}}}\left(x_{2}\right) \\
& \times \frac{\int_{X} \chi\left(x_{3}\right) e^{w_{1} w_{2} x_{3} t} d \mu_{\xi^{w_{1} w_{2}}}\left(x_{3}\right)}{\int_{X} e^{d w_{1} w_{2} w_{3} x_{4} t} d \mu_{\xi^{d w_{1} w_{2} w_{3}}}\left(x_{4}\right)}
\end{aligned}
$$

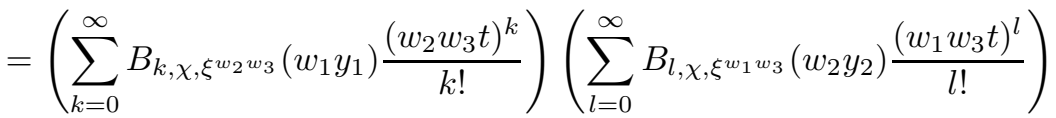

$$
\begin{aligned}
& \times\left(\sum_{m=0}^{\infty} S_{m}\left(d w_{3}-1 ; \chi, \xi^{w_{1} w_{2}}\right) \frac{\left(w_{1} w_{2} t\right)^{m}}{m !}\right) \\
& =\sum_{n=0}^{\infty}\left(\sum_{k+l+m=n}\left(\begin{array}{c}
n \\
k, l, m
\end{array}\right) B_{k, \chi, \xi^{w_{2} w_{3}}}\left(w_{1} y_{1}\right) B_{l, \chi, \xi^{w_{1} w_{3}}}\left(w_{2} y_{2}\right) S_{m}\left(d w_{3}-1 ; \chi, \xi^{w_{1} w_{2}}\right)\right. \\
& \left.\times w_{1}^{l+m} w_{2}^{k+m} w_{3}^{k+l}\right) \frac{t^{n}}{n !} .
\end{aligned}
$$

(2) Invoking (9), (20) can also be written as

$$
\begin{aligned}
& I\left(\Lambda_{23}^{1}\right)=\sum_{a=0}^{d w_{3}-1} \chi(a) \xi^{a w_{1} w_{2}} \int_{X} \chi\left(x_{1}\right) t e^{w_{2} w_{3}\left(x_{1}+w_{1} y_{1}\right) t} d \mu_{\xi^{w_{2} w_{3}}}\left(x_{1}\right) \\
& \times \int_{X} \chi\left(x_{2}\right) t e^{w_{1} w_{3}\left(x_{2}+w_{2} y_{2}+\frac{w_{2}}{w_{3}} a\right) t} d \mu_{\xi^{w_{1} w_{3}}}\left(x_{2}\right)
\end{aligned}
$$

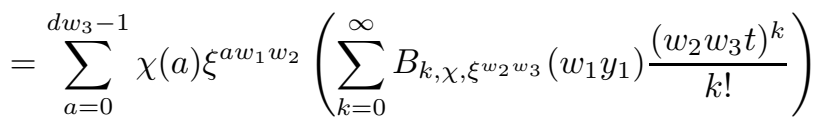

$$
\begin{aligned}
& \times\left(\sum_{l=0}^{\infty} B_{l, \chi, \xi^{w_{1} w_{3}}}\left(w_{2} y_{2}+\frac{w_{2}}{w_{3}} a\right) \frac{\left(w_{1} w_{3} t\right)^{l}}{l !}\right)
\end{aligned}
$$

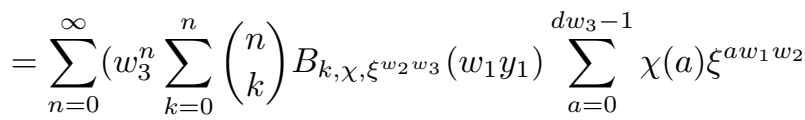




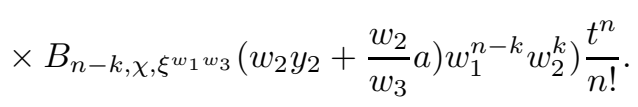

(a-2) Here we write $I\left(\Lambda_{23}^{2}\right)$ in three different ways:

(1)

$$
\begin{aligned}
& I\left(\Lambda_{23}^{2}\right)=\int_{X} \chi\left(x_{1}\right) t e^{w_{2} w_{3}\left(x_{1}+w_{1} y_{1}\right) t} d \mu_{\xi^{w_{2} w_{3}}}\left(x_{1}\right)
\end{aligned}
$$

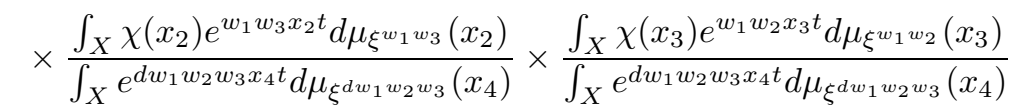

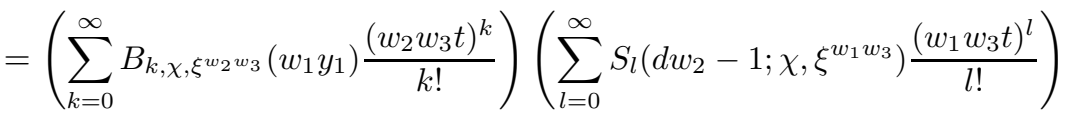

$$
\begin{aligned}
& \times\left(\sum_{m=0}^{\infty} S_{m}\left(d w_{3}-1 ; \chi, \xi^{w_{1} w_{2}}\right) \frac{\left(w_{1} w_{2} t\right)^{m}}{m !}\right)
\end{aligned}
$$

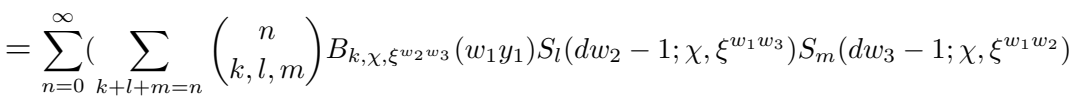

$$
\begin{aligned}
& \left.\times w_{1}^{l+m} w_{2}^{k+m} w_{3}^{k+l}\right) \frac{t^{n}}{n !} .
\end{aligned}
$$

(2) Invoking (9), (29) can also be written as

$$
\begin{aligned}
& I\left(\Lambda_{23}^{2}\right)=\sum_{a=0}^{d w_{2}-1} \chi(a) \xi^{a w_{1} w_{3}} \int_{X} \chi\left(x_{1}\right) t e^{w_{2} w_{3}\left(x_{1}+w_{1} y_{1}+\frac{w_{1}}{w_{2}} a\right) t} d \mu_{\xi^{w_{2} w_{3}}}\left(x_{1}\right) \\
& \times \frac{\int_{X} \chi\left(x_{3}\right) e^{w_{1} w_{2} x_{3} t} d \mu_{\xi^{w_{1} w_{2}}}\left(x_{3}\right)}{\int_{X} e^{d w_{1} w_{2} w_{3} x_{4} t} d \mu_{\xi^{d w_{1} w_{2} w_{3}}\left(x_{4}\right)}}
\end{aligned}
$$

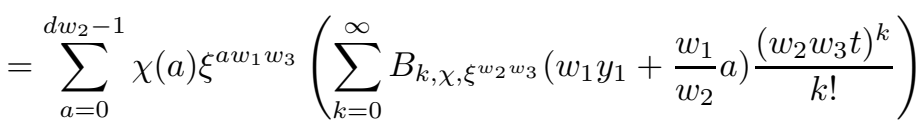

$$
\begin{aligned}
& \times\left(\sum_{l=0}^{\infty} S_{l}\left(d w_{3}-1 ; \chi, \xi^{w_{1} w_{2}}\right) \frac{\left(w_{1} w_{2} t\right)^{l}}{l !}\right) \\
& =\sum_{n=0}^{\infty}\left(w_{2}^{n} \sum_{k=0}^{n}\left(\begin{array}{l}
n \\
k
\end{array}\right) \sum_{a=0}^{d w_{2}-1} \chi(a) \xi^{a w_{1} w_{3}} B_{k, \chi, \xi^{w_{2} w_{3}}}\left(w_{1} y_{1}+\frac{w_{1}}{w_{2}} a\right)\right. \\
& \left.\times S_{n-k}\left(d w_{3}-1 ; \chi, \xi^{w_{1} w_{2}}\right) w_{1}^{n-k} w_{3}^{k}\right) \frac{t^{n}}{n !} .
\end{aligned}
$$

(3) Invoking (9) once again, (31) can be written as

$$
\begin{aligned}
& I\left(\Lambda_{23}^{2}\right)=\sum_{a=0}^{d w_{2}-1} \chi(a) \xi^{a w_{1} w_{3}} \sum_{b=0}^{d w_{3}-1} \chi(b) \xi^{b w_{1} w_{2}} \int_{X} \chi\left(x_{1}\right) t e^{w_{2} w_{3}\left(x_{1}+w_{1} y_{1}+\frac{w_{1}}{w_{2}} a+\frac{w_{1}}{w_{3}} b\right) t} d \mu_{\xi_{2} w_{2} w_{3}}\left(x_{1}\right) \\
& =\sum_{a=0}^{d w_{2}-1} \chi(a) \xi^{a w_{1} w_{3}} \sum_{b=0}^{d w_{3}-1} \chi(b) \xi^{\xi w_{1} w_{2}}\left(\sum_{n=0}^{\infty} B_{n, \chi, \xi^{w} w_{2} w_{3}}\left(w_{1} y_{1}+\frac{w_{1}}{w_{2}} a+\frac{w_{1}}{w_{3}} b\right) \frac{\left(w_{2} w_{3} t\right)^{n}}{n !}\right)
\end{aligned}
$$




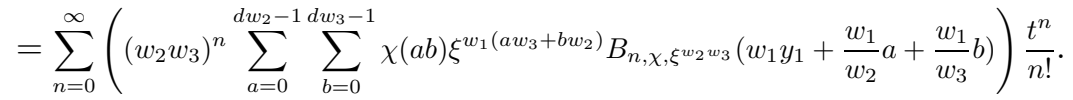

$$
\begin{aligned}
& I\left(\Lambda_{23}^{3}\right)=\frac{\int_{X} \chi\left(x_{1}\right) e^{w_{2} w_{3} x_{1} t} d \mu_{\xi^{w_{2} w_{3}}}\left(x_{1}\right)}{\int_{X} e^{d w_{1} w_{2} w_{3} x_{4} t} d \mu_{\xi^{d w_{1} w_{2} w_{3}}}\left(x_{4}\right)} \times \frac{\int_{X} \chi\left(x_{2}\right) e^{w_{1} w_{3} x_{2} t} d \mu_{\xi^{w_{1} w_{3}}}\left(x_{2}\right)}{\int_{X} e^{d w_{1} w_{2} w_{3} x_{4} t} d \mu_{\xi^{d w_{1} w_{2} w_{3}}\left(x_{4}\right)}} \\
& \times \frac{\int_{X} \chi\left(x_{3}\right) e^{w_{1} w_{2} x_{3} t} d \mu_{\xi^{w_{1} w_{2}}}\left(x_{3}\right)}{\int_{X} e^{d w_{1} w_{2} w_{3} x_{4} t} d \mu_{\xi^{d w_{1} w_{2} w_{3}}}\left(x_{4}\right)} \\
& =\left(\sum_{k=0}^{\infty} S_{k}\left(d w_{1}-1 ; \chi, \xi^{w_{2} w_{3}}\right) \frac{\left(w_{2} w_{3} t\right)^{k}}{k !}\right)\left(\sum_{l=0}^{\infty} S_{l}\left(d w_{2}-1 ; \chi, \xi^{w_{1} w_{3}}\right) \frac{\left(w_{1} w_{3} t\right)^{l}}{l !}\right) \\
& \times\left(\sum_{m=0}^{\infty} S_{m}\left(d w_{3}-1 ; \chi, \xi^{w_{1} w_{2}}\right) \frac{\left(w_{1} w_{2} t\right)^{m}}{m !}\right) \\
& =\sum_{n=0}^{\infty}\left(\sum_{k+l+m=n}\left(\begin{array}{c}
n \\
k, l, m
\end{array}\right) S_{k}\left(d w_{1}-1 ; \chi, \xi^{w_{2} w_{3}}\right) S_{l}\left(d w_{2}-1 ; \chi, \xi^{w_{1} w_{3}}\right)\right. \\
& \left.\times S_{m}\left(d w_{3}-1 ; \chi, \xi^{w_{1} w_{2}}\right) w_{1}^{l+m} w_{2}^{k+m} w_{3}^{k+l}\right) \frac{t^{n}}{n !} .
\end{aligned}
$$

(b) For Type $\Lambda_{13}^{i}(i=0,1,2,3)$, we may consider the analogous things to the ones in (a-0), (a-1), (a-2), and (a-3). However, each of those can be obtained from the corresponding ones in (a-0), (a-1), (a-2), and (a-3). Indeed, if we substitute $w_{2} w_{3}, w_{1} w_{3}, w_{1} w_{2}$ respectively for $w_{1}, w_{2}, w_{3}$ in $\frac{I\left(\Lambda_{23}^{i}\right)}{t^{3-i}}$ (cf. (13)), this amounts to replacing $t$ by $w_{1} w_{2} w_{3} t$ and $\xi$ by $\xi^{w_{1} w_{2} w_{3}}$ in $\frac{I\left(\Lambda_{13}^{i}\right)}{t^{3-i}}$ (cf. (15)). So, upon replacing $w_{1}, w_{2}, w_{3}$ respectively by $w_{2} w_{3}, w_{1} w_{3}, w_{1} w_{2}$, dividing by $\left(w_{1} w_{2} w_{3}\right)^{n}$, and replacing $\xi^{w_{1} w_{2} w_{3}}$ by $\xi$, in each of the expressions of (25), (27), (28), (30), (32)-(34), we will get the corresponding symmetric identities for Type $\Lambda_{13}^{i}(i=0,1,2,3)$.

(c-0)

$$
\begin{aligned}
& I\left(\Lambda_{12}^{0}\right)=\int_{X} \chi\left(x_{1}\right) t e^{w_{1}\left(x_{1}+w_{2} y\right) t} d \mu_{\xi^{w_{1}}}\left(x_{1}\right) \int_{X} \chi\left(x_{2}\right) t e^{w_{2}\left(x_{2}+w_{3} y\right) t} d \mu_{\xi^{w_{2}}}\left(x_{2}\right) \\
& \times \int_{X} \chi\left(x_{3}\right) t e^{w_{3}\left(x_{3}+w_{1} y\right) t} d \mu_{\xi^{w_{3}}}\left(x_{3}\right) \\
& =\left(\sum_{k=0}^{\infty} \frac{B_{k, \chi, \xi^{w_{1}}}\left(w_{2} y\right)}{k !}\left(w_{1} t\right)^{k}\right)\left(\sum_{l=0}^{\infty} \frac{B_{l, \chi, \xi^{w_{2}}}\left(w_{3} y\right)}{l !}\left(w_{2} t\right)^{l}\right)\left(\sum_{m=0}^{\infty} \frac{B_{m, \chi, \xi^{w_{3}}}\left(w_{1} y\right)}{m !}\left(w_{3} t\right)^{m}\right) \\
& (35) \quad=\sum_{n=0}^{\infty}\left(\sum_{k+l+m=n}\left(\begin{array}{c}
n \\
k, l, m
\end{array}\right) B_{k, \chi, \xi^{w_{1}}}\left(w_{2} y\right) B_{l, \chi, \xi^{w_{2}}}\left(w_{3} y\right) B_{m, \chi, \xi^{w_{3}}}\left(w_{1} y\right) w_{1}^{k} w_{2}^{l} w_{3}^{m}\right) \frac{t^{n}}{n !} \text {. }
\end{aligned}
$$

$I\left(\Lambda_{12}^{1}\right)=\frac{\int_{X} \chi\left(x_{1}\right) e^{w_{1} x_{1} t} d \mu_{\xi^{w_{1}}}\left(x_{1}\right)}{\int_{X} e^{d w_{1} w_{2} z_{3} t} d \mu_{\xi^{d w_{1} w_{2}}}\left(z_{3}\right)} \times \frac{\int_{X} \chi\left(x_{2}\right) e^{w_{2} x_{2} t} d \mu_{\xi^{w_{2}}}\left(x_{2}\right)}{\int_{X} e^{d w_{2} w_{3} z_{1} t} d \mu_{\xi^{d w_{2} w_{3}}}\left(z_{1}\right)} \times \frac{\int_{X} \chi\left(x_{3}\right) e^{w_{3} x_{3} t} d \mu_{\xi^{w_{3}}}\left(x_{3}\right)}{\int_{X} e^{d w_{3} w_{1} z_{2} t} d \mu_{\xi^{d w_{3} w_{1}}}\left(z_{2}\right)}$ 


$$
\begin{aligned}
= & \left(\sum_{k=0}^{\infty} S_{k}\left(d w_{2}-1 ; \chi, \xi^{w_{1}}\right) \frac{\left(w_{1} t\right)^{k}}{k !}\right)\left(\sum_{l=0}^{\infty} S_{l}\left(d w_{3}-1 ; \chi, \xi^{w_{2}}\right) \frac{\left(w_{2} t\right)^{l}}{l !}\right) \\
& \times\left(\sum_{m=0}^{\infty} S_{m}\left(d w_{1}-1 ; \chi, \xi^{w_{3}}\right) \frac{\left(w_{3} t\right)^{m}}{m !}\right) \\
= & \sum_{n=0}^{\infty}\left(\sum_{k+l+m=n}\left(\begin{array}{c}
n \\
k, l, m
\end{array}\right) S_{k}\left(d w_{2}-1 ; \chi, \xi^{w_{1}}\right) S_{l}\left(d w_{3}-1 ; \chi, \xi^{w_{2}}\right)\right. \\
& \left.\times S_{m}\left(d w_{1}-1 ; \chi, \xi^{w_{3}}\right) w_{1}^{k} w_{2}^{l} w_{3}^{m}\right) \frac{t^{n}}{n !} .
\end{aligned}
$$

\section{Main theorems}

As we noted earlier, the various types of quotients of $p$-adic integrals in Section 2 and Section 3 are respectively invariant under the transposition of $w_{1}, w_{2}$ and any permutation of $w_{1}, w_{2}, w_{3}$. So the corresponding expressions in Section 4 and Section 5 are respectively also invariant under the transposition of $w_{1}, w_{2}$ and any permutation of $w_{1}, w_{2}, w_{3}$. Thus our results about identities of symmetry will be immediate consequences of this observation.

However, not all permutations of an expression in Section 5 yield distinct ones. In fact, as these expressions are obtained by permuting $w_{1}, w_{2}, w_{3}$ in a single one labeled by them, they can be viewed as a group in a natural manner and hence it is isomorphic to a quotient of $S_{3}$. In particular, the number of possible distinct expressions are 1, 2, 3, or 6. (a-0), (a-1(1)), (a-1(2)), and (a$2(2))$ give the full six identities of symmetry, (a-2(1)) and (a-2(3)) yield three identities of symmetry, and (c-0) and (c-1) give two identities of symmetry, while the expression in (a-3) yields no identities of symmetry. Similarly, $(\alpha-$ $0),(\alpha-1(1))$, and $(\alpha-1(2))$ give two identities of symmetry but $(\alpha-2)$ yields no identity of symmetry.

Here we will just consider the cases of Theorems 7 and 11, leaving the others as easy exercises for the reader. As for the case of Theorem 7, in addition to (50)-(52), we get the following three ones:

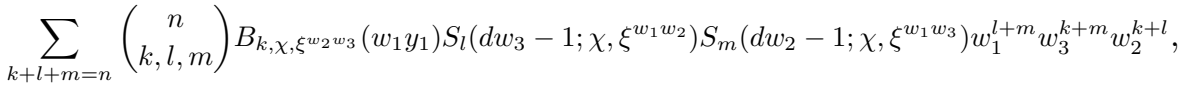

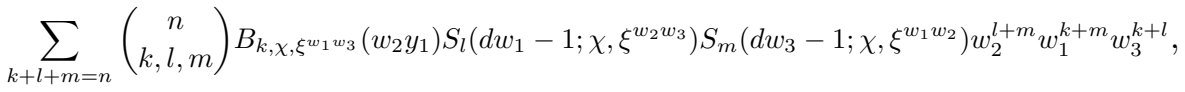

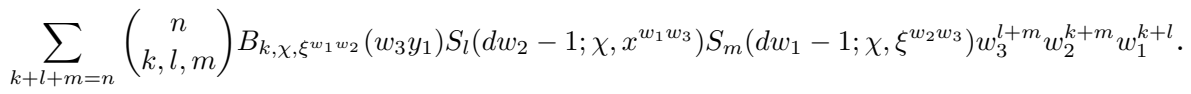

But, by interchanging $l$ and $m$, we see that (37), (38), and (39) are respectively equal to (50), (51), and (52). As to Theorem 11, in addition to (56) and 
(57), we have:

(40) $\sum_{k+l+m=n}\left(\begin{array}{c}n \\ k, l, m\end{array}\right) S_{k}\left(d w_{2}-1 ; \chi, \xi^{w_{1}}\right) S_{l}\left(d w_{3}-1 ; \chi, \xi^{w_{2}}\right) S_{m}\left(d w_{1}-1 ; \chi, \xi^{w_{3}}\right) w_{1}^{k} w_{2}^{l} w_{3}^{m}$,

(41) $\sum_{k+l+m=n}\left(\begin{array}{c}n \\ k, l, m\end{array}\right) S_{k}\left(d w_{3}-1 ; \chi, \xi^{w_{2}}\right) S_{l}\left(d w_{1}-1 ; \chi, \xi^{w_{3}}\right) S_{m}\left(d w_{2}-1 ; \chi, \xi^{w_{1}}\right) w_{2}^{k} w_{3}^{l} w_{1}^{m}$,

(42) $\sum_{k+l+m=n}\left(\begin{array}{c}n \\ k, l, m\end{array}\right) S_{k}\left(d w_{3}-1 ; \chi, \xi^{w_{1}}\right) S_{l}\left(d w_{2}-1 ; \chi, \xi^{w_{3}}\right) S_{m}\left(d w_{1}-1 ; \chi, \xi^{w_{2}}\right) w_{1}^{k} w_{3}^{l} w_{2}^{m}$,

(43) $\sum_{k+l+m=n}\left(\begin{array}{c}n \\ k, l, m\end{array}\right) S_{k}\left(d w_{2}-1 ; \chi, \xi^{w_{3}}\right) S_{l}\left(d w_{1}-1 ; \chi, \xi^{w_{2}}\right) S_{m}\left(d w_{3}-1 ; \chi, \xi^{w_{1}}\right) w_{3}^{k} w_{2}^{l} w_{1}^{m}$.

However, (40) and (41) are equal to (56), as we can see by applying the permutations $k \rightarrow l, l \rightarrow m, m \rightarrow k$ for (40) and $k \rightarrow m, l \rightarrow k, m \rightarrow l$ for (41). Similarly, we see that (42) and (43) are equal to (57), by applying permutations $k \rightarrow l, l \rightarrow m, m \rightarrow k$ for (42) and $k \rightarrow m, l \rightarrow k, m \rightarrow l$ for (43).

Theorem 1. Let $w_{1}, w_{2}$ be any positive integers, such that $r$ does not divide $w_{1}, w_{2}$. Then we have:

$$
\begin{aligned}
& \sum_{k=0}^{n}\left(\begin{array}{l}
n \\
k
\end{array}\right) B_{k, \chi, \xi^{w_{1}}}\left(w_{2} y_{1}\right) B_{n-k, \chi, \xi^{w_{2}}}\left(w_{1} y_{2}\right) w_{1}^{k} w_{2}^{n-k} \\
= & \sum_{k=0}^{n}\left(\begin{array}{l}
n \\
k
\end{array}\right) B_{k, \chi, \xi^{w_{2}}}\left(w_{1} y_{1}\right) B_{n-k, \chi, \xi^{w_{1}}}\left(w_{2} y_{2}\right) w_{2}^{k} w_{1}^{n-k} .
\end{aligned}
$$

Theorem 2. Let $w_{1}, w_{2}$ be any positive integers, such that $r$ does not divide $w_{1} w_{2}$. Then we have:

$$
\begin{aligned}
& \sum_{k=0}^{n}\left(\begin{array}{l}
n \\
k
\end{array}\right) B_{k, \chi, \xi^{w_{1}}}\left(w_{2} y_{1}\right) S_{n-k}\left(d w_{1}-1 ; \chi, \xi^{w_{2}}\right) w_{1}^{k} w_{2}^{n-k} \\
= & \sum_{k=0}^{n}\left(\begin{array}{l}
n \\
k
\end{array}\right) B_{k, \chi, \xi^{w_{2}}}\left(w_{1} y_{1}\right) S_{n-k}\left(d w_{2}-1 ; \chi, \xi^{w_{1}}\right) w_{2}^{k} w_{1}^{n-k} .
\end{aligned}
$$

Theorem 3. Let $w_{1}, w_{2}$ be any positive integers, such that $r$ does not divide $w_{1} w_{2}$. Then we have:

$$
\begin{aligned}
& w_{1}^{n} \sum_{a=0}^{d w_{1}-1} \chi(a) \xi^{a w_{2}} B_{n, \chi, \xi^{w_{1}}}\left(w_{2} y_{1}+\frac{w_{2}}{w_{1}} a\right) \\
= & w_{2}^{n} \sum_{a=0}^{d w_{2}-1} \chi(a) \xi^{a w_{1}} B_{n, \chi, \xi^{w_{2}}}\left(w_{1} y_{1}+\frac{w_{1}}{w_{2}} a\right) .
\end{aligned}
$$


Theorem 4. Let $w_{1}, w_{2}, w_{3}$ be any positive integers, such that $r$ does not divide $w_{2} w_{3}, w_{1} w_{3}, w_{1} w_{2}$. Then we have:

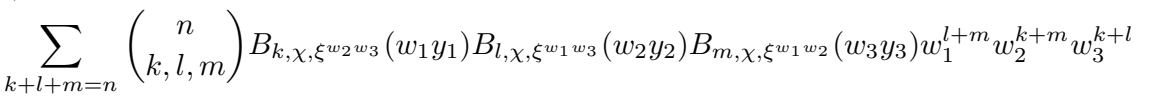

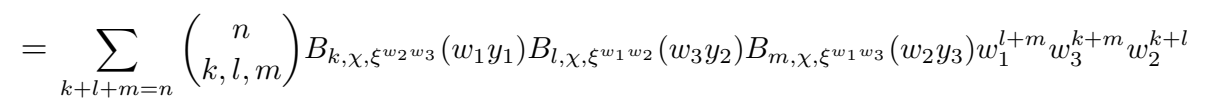

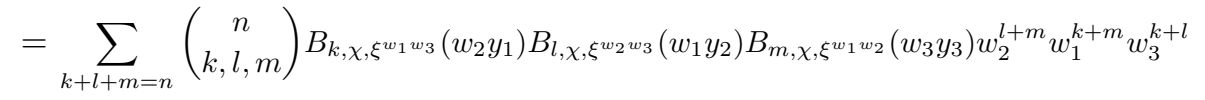

$$
\begin{aligned}
& =\sum_{k+l+m=n}\left(\begin{array}{c}
n \\
k, l, m
\end{array}\right) B_{k, \chi, \xi^{w_{1} w_{3}}}\left(w_{2} y_{1}\right) B_{l, \chi, \xi^{w_{1} w_{2}}}\left(w_{3} y_{2}\right) B_{m, \chi, \xi^{w_{2} w_{3}}}\left(w_{1} y_{3}\right) w_{2}^{l+m} w_{3}^{k+m} w_{1}^{k+l}
\end{aligned}
$$

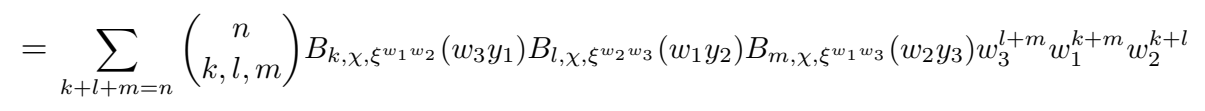

$$
\begin{aligned}
& =\sum_{k+l+m=n}\left(\begin{array}{c}
n \\
k, l, m
\end{array}\right) B_{k, \chi, \xi^{w_{1} w_{2}}}\left(w_{3} y_{1}\right) B_{l, \chi, \xi^{w_{1} w_{3}}}\left(w_{2} y_{2}\right) B_{m, \chi, \xi^{w_{2} w_{3}}}\left(w_{1} y_{3}\right) w_{3}^{l+m} w_{2}^{k+m} w_{1}^{k+l} \text {. }
\end{aligned}
$$

Theorem 5. Let $w_{1}, w_{2}, w_{3}$ be any positive integers, such that $r$ does not divide $w_{1} w_{2} w_{3}$. Then we have:

(48)

$$
\begin{aligned}
& \sum_{k+l+m=n}\left(\begin{array}{c}
n \\
k, l, m
\end{array}\right) B_{k, \chi, \xi^{w_{2} w_{3}}\left(w_{1} y_{1}\right)} B_{l, \chi, \xi^{w_{1} w_{3}}}\left(w_{2} y_{2}\right) S_{m}\left(d w_{3}-1 ; \chi, \xi^{w_{1} w_{2}}\right) w_{1}^{l+m} w_{2}^{k+m} w_{3}^{k+l}
\end{aligned}
$$

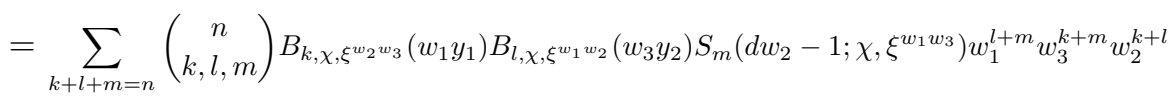

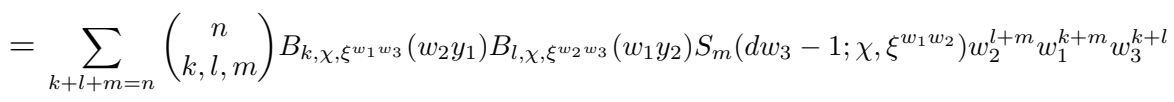

$$
\begin{aligned}
& =\sum_{k+l+m=n}\left(\begin{array}{c}
n \\
k, l, m
\end{array}\right) B_{k, \chi, \xi^{w_{1} w_{3}}}\left(w_{2} y_{1}\right) B_{l, \chi, \xi^{w_{1} w_{2}}}\left(w_{3} y_{2}\right) S_{m}\left(d w_{1}-1 ; \chi, \xi^{w_{2} w_{3}}\right) w_{2}^{l+m} w_{3}^{k+m} w_{1}^{k+l}
\end{aligned}
$$

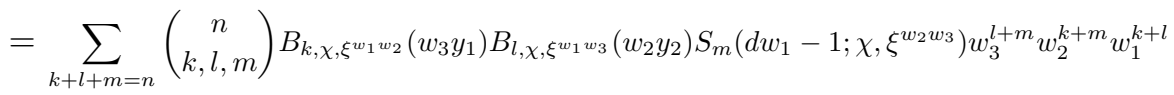

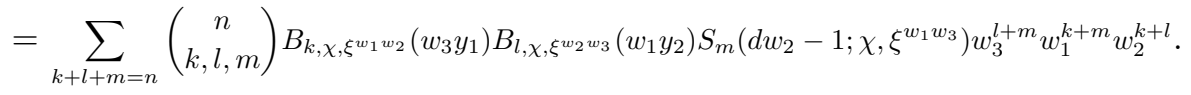

Theorem 6. Let $w_{1}, w_{2}, w_{3}$ be any positive integers, such that $r$ does not divide $w_{1} w_{2} w_{3}$. Then we have:

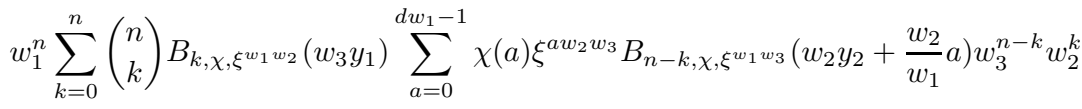

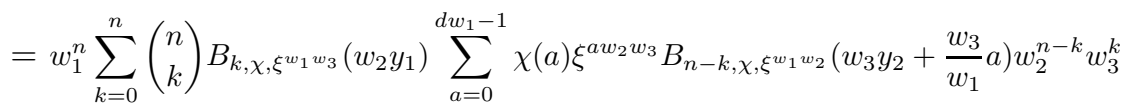




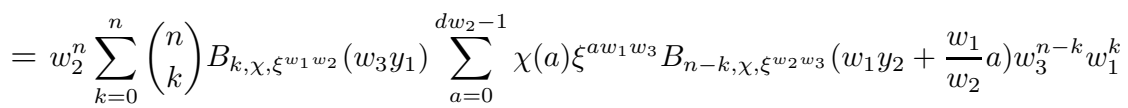

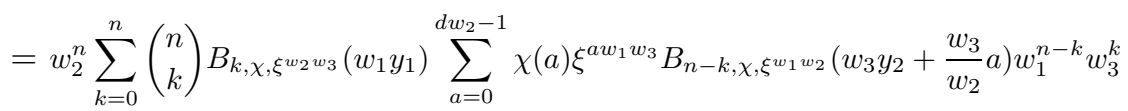

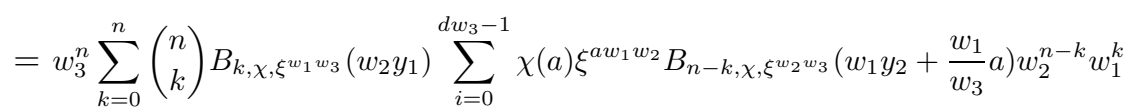

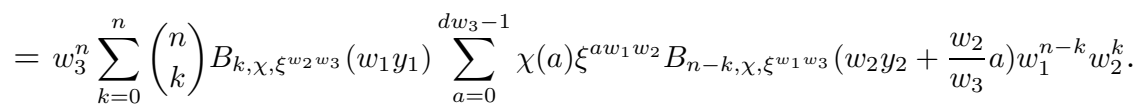

Theorem 7. Let $w_{1}, w_{2}, w_{3}$ be any positive integers, such that $r$ does not divide $w_{1} w_{2} w_{3}$. Then we have the following three symmetries in $w_{1}, w_{2}, w_{3}$ :

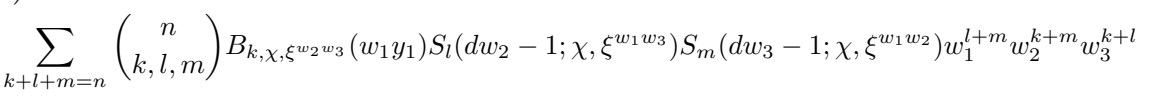

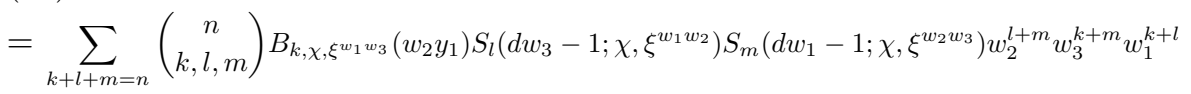

$=\sum_{k+l+m=n}\left(\begin{array}{c}n \\ k, l, m\end{array}\right) B_{k, \chi, \xi^{w_{1} w_{2}}}\left(w_{3} y_{1}\right) S_{l}\left(d w_{1}-1 ; \chi, \xi^{w_{2} w_{3}}\right) S_{m}\left(d w_{2}-1 ; \chi, \xi^{w_{1} w_{3}}\right) w_{3}^{l+m} w_{1}^{k+m} w_{2}^{k+l}$.

Theorem 8. Let $w_{1}, w_{2}, w_{3}$ be any positive integers, such that $r$ does not divide $w_{1} w_{2} w_{3}$. Then we have:

$$
\begin{aligned}
& w_{1}^{n} \sum_{k=0}^{n}\left(\begin{array}{l}
n \\
k
\end{array}\right) \sum_{a=0}^{d w_{1}-1} \chi(a) \xi^{a w_{2} w_{3}} B_{k, \chi, \xi^{a w_{1} w_{3}}}\left(w_{2} y_{1}+\frac{w_{2}}{w_{1}} a\right) S_{n-k}\left(d w_{3}-1 ; \chi, \xi^{w_{1} w_{2}}\right) w_{2}^{n-k} w_{3}^{k} \\
& =w_{1}^{n} \sum_{k=0}^{n}\left(\begin{array}{l}
n \\
k
\end{array}\right) \sum_{a=0}^{d w_{1}-1} \chi(a) \xi^{a w_{2} w_{3}} B_{k, \chi, \xi^{w_{1} w_{2}}}\left(w_{3} y_{1}+\frac{w_{3}}{w_{1}} a\right) S_{n-k}\left(d w_{2}-1 ; \chi, \xi^{w_{1} w_{3}}\right) w_{3}^{n-k} w_{2}^{k} \\
& =w_{2}^{n} \sum_{k=0}^{n}\left(\begin{array}{l}
n \\
k
\end{array}\right) \sum_{a=0}^{d w_{2}-1} \chi(a) \xi^{a w_{1} w_{3}} B_{k, \chi, \xi^{w_{2} w_{3}}}\left(w_{1} y_{1}+\frac{w_{1}}{w_{2}} a\right) S_{n-k}\left(d w_{3}-1 ; \chi, \xi^{w_{1} w_{2}}\right) w_{1}^{n-k} w_{3}^{k} \\
& =w_{2}^{n} \sum_{k=0}^{n}\left(\begin{array}{l}
n \\
k
\end{array}\right) \sum_{a=0}^{d w_{2}-1} \chi(a) \xi^{a w_{1} w_{3}} B_{k, \chi, \xi^{w_{1} w_{2}}}\left(w_{3} y_{1}+\frac{w_{3}}{w_{2}} a\right) S_{n-k}\left(d w_{1}-1 ; \chi, x^{w_{2} w_{3}}\right) w_{3}^{n-k} w_{1}^{k} \\
& =w_{3}^{n} \sum_{k=0}^{n}\left(\begin{array}{l}
n \\
k
\end{array}\right) \sum_{a=0}^{d w_{3}-1} \chi(a) \xi^{a w_{1} w_{2}} B_{k, \chi, \xi^{w_{2} w_{3}}}\left(w_{1} y_{1}+\frac{w_{1}}{w_{3}} a\right) S_{n-k}\left(d w_{2}-1 ; \chi, \xi^{w_{1} w_{3}}\right) w_{1}^{n-k} w_{2}^{k} \\
& =w_{3}^{n} \sum_{k=0}^{n}\left(\begin{array}{l}
n \\
k
\end{array}\right) \sum_{a=0}^{d w_{3}-1} \chi(a) \xi^{a w_{1} w_{2}} B_{k, \chi, \xi^{w_{1} w_{3}}}\left(w_{2} y_{1}+\frac{w_{2}}{w_{3}} a\right) S_{n-k}\left(d w_{1}-1 ; \chi, \xi^{w_{2} w_{3}}\right) w_{2}^{n-k} w_{1}^{k} \text {. }
\end{aligned}
$$


Theorem 9. Let $w_{1}, w_{2}, w_{3}$ be any positive integers, such that $r$ does not divide $w_{1} w_{2} w_{3}$. Then we have the following three symmetries in $w_{1}, w_{2}, w_{3}$ :

$$
\begin{aligned}
& \text { (54) } \quad\left(w_{1} w_{2}\right)^{n} \sum_{a=0}^{d w_{1}-1} \sum_{b=0}^{d w_{2}-1} \chi(a b) \xi^{w_{3}\left(a w_{2}+b w_{1}\right)} B_{n, \chi, \xi^{w_{1} w_{2}}}\left(w_{3} y_{1}+\frac{w_{3}}{w_{1}} a+\frac{w_{3}}{w_{2}} b\right) \\
& =\left(w_{2} w_{3}\right)^{n} \sum_{a=0}^{d w_{2}-1} \sum_{b=0}^{d w_{3}-1} \chi(a b) \xi^{w_{1}\left(a w_{3}+b w_{2}\right)} B_{n, \chi, \xi^{w_{2} w_{3}}}\left(w_{1} y_{1}+\frac{w_{1}}{w_{2}} a+\frac{w_{1}}{w_{3}} b\right) \\
& =\left(w_{3} w_{1}\right)^{n} \sum_{a=0}^{d w_{3}-1} \sum_{b=0}^{d w_{1}-1} \chi(a b) \xi^{w_{2}\left(a w_{1}+b w_{3}\right)} B_{n, \chi, \xi^{w_{1} w_{3}}}\left(w_{2} y_{1}+\frac{w_{2}}{w_{3}} a+\frac{w_{2}}{w_{1}} b\right) .
\end{aligned}
$$

Theorem 10. Let $w_{1}, w_{2}, w_{3}$ be any positive integers, such that $r$ does not divide $w_{1}, w_{2}, w_{3}$. Then we have the following two symmetries in symmetries in $w_{1}, w_{2}, w_{3}$ :

$$
\begin{aligned}
& \sum_{k+l+m=n}\left(\begin{array}{c}
n \\
k, l, m
\end{array}\right) B_{k, \chi, \xi^{w_{3}}}\left(w_{1} y\right) B_{l, \chi, \xi^{w_{1}}}\left(w_{2} y\right) B_{m, \chi, \xi^{w_{2}}}\left(w_{3} y\right) w_{3}^{k} w_{1}^{l} w_{2}^{m} \\
= & \sum_{k+l+m=n}\left(\begin{array}{c}
n \\
k, l, m
\end{array}\right) B_{k, \chi, \xi^{w_{2}}}\left(w_{1} y\right) B_{l, \chi, \xi^{w_{1}}}\left(w_{3} y\right) B_{m, \chi, \xi^{w_{3}}}\left(w_{2} y\right) w_{2}^{k} w_{1}^{l} w_{3}^{m} .
\end{aligned}
$$

Theorem 11. Let $w_{1}, w_{2}, w_{3}$ be any positive integers, such that $r$ does not divide $w_{2} w_{3}, w_{1} w_{3}, w_{1} w_{2}$. Then we have the following two symmetries in $w_{1}$, $w_{2}, w_{3}$ :

$$
\sum_{k+l+m=n}\left(\begin{array}{c}
n \\
k, l, m
\end{array}\right) S_{k}\left(d w_{1}-1 ; \chi, \xi^{w_{3}}\right) S_{l}\left(d w_{2}-1 ; \chi, \xi^{w_{1}}\right) S_{m}\left(d w_{3}-1 ; \chi, \xi^{w_{2}}\right) w_{3}^{k} w_{1}^{l} w_{2}^{m}
$$

$$
=\sum_{k+l+m=n}\left(\begin{array}{c}
n \\
k, l, m
\end{array}\right) S_{k}\left(d w_{1}-1 ; \chi, \xi^{w_{2}}\right) S_{l}\left(d w_{3}-1 ; \chi, \xi^{w_{1}}\right) S_{m}\left(d w_{2}-1 ; \chi, \xi^{w_{3}}\right) w_{2}^{k} w_{1}^{l} w_{3}^{m} .
$$

\section{References}

[1] A. Bayad and T. Kim, Identities for the Bernoulli, the Euler and the Genocchi numbers and polynomials, Adv. Stud. Contemp. Math. (Kyungshang) 20 (2010), no. 2, 247-253.

[2] E. Deeba and D. Rodriguez, Stirling's and Bernoulli numbers, Amer. Math. Monthly 98 (1991), no. 5, 423-426.

[3] F. T. Howard, Applications of a recurrence for the Bernoulli numbers, J. Number Theory 52 (1995), no. 1, 157-172.

[4] D. S. Kim, Identities of symmetry for q-Bernoulli polynomials, Comput. Math. Appl. 60 (2010), no. 8, 2350-2359.

[5] _ Symmetry identities for generalized twisted Euler polynomials twisted by unramified roots of unity, Proc. Jangjeon Math. Soc. 15 (2012), no. 3, 303-316.

[6] - Identities of symmetry for generalized twisted Bernoulli polynomials twisted by ramified roots of unity, An. Stiint. Univ. Al. I. Cuza Iasi, Ser. Noua, Mat. 60 (2014), no. 1, 19-36. 
[7] D. S. Kim, N. Lee, J. Na, and K. H. Park, Identities of symmetry for higher-order Euler polynomials in three variables (I), Adv. Stud. Contemp. Math. (Kyungshang) 22 (2012), no. $1,51-74$.

[8] — Abundant symmetry for higher-order Bernoulli polynomials (I), Adv. Stud. Contemp. Math. (Kyungshang) 23 (2013), no. 3, 461-482.

[9] D. S. Kim and K. H. Park, Identities of symmetry for Euler polynomials arising from quotients of fermionic integrals invariant under $S_{3}$, J. Inequal. Appl. 2010 (2010), Article ID 851521, 16 pages.

[10] - Identities of symmetry for Bernoulli polynomials arising from quotients of Volkenborn integrals invariant under $S_{3}$, Appl. Math. Comput. 219 (2013), no. 10, 5096-5104.

[11] T. Kim, Symmetry p-adic invariant integral on $\mathbb{Z}_{p}$ for Bernoulli and Euler polynomials, J. Difference Equ. Appl. 14 (2008), no. 12, 1267-1277.

[12] $\longrightarrow$ On the symmetries of the q-Bernoulli polynomials, Abstr. Appl. Anal. 2008 (2008), Art. ID 914367, 7 pp.

[13] — On the symmetric properties for the generalized twisted Bernoulli polynomials, J. Inequal. Appl. 2009 (2009), Article ID 164743, 8 pages.

[14] - Symmetry of power sum polynomials and multivariate fermionic p-adic invariant integral on $\mathbb{Z}_{p}$, Russ. J. Math. Phys. 16 (2009), no. 1, 93-96.

[15] T. Kim, L.-C. Jang, Y.-H. Kim, and K.-W. Hwang, On the identities of symmetry for the generalized Bernoulli polynomials attached to of higher order, J. Inequal. Appl. 2009 (2009), Art. ID 640152, 7 pp.

[16] T. Kim, S.-H. Rim, and B. Lee, Some identities of symmetry for the generalized Bernoulli numbers and polynomials, Abstr. Appl. Anal. 2009 (2009), Art. ID 848943, 8 pp.

[17] Y.-H. Kim and K.-W. Hwang, Symmetry of power sum and twisted Bernoulli polynomials, Adv. Stud. Contemp. Math. (Kyungshang) 18 (2009), no. 2, 127-133.

[18] N. Koblitz, A new proof of certain formulas for p-adic L-functions, Duke Math. J. 46 (1979), no. 2, 455-468.

[19] S.-H. Rim, Y.-H. Kim, B. Lee, and T. Kim, Some identities of the generalized twisted Bernoulli numbers and polynomials of higher order, J. Comput. Anal. Appl. 12 (2010), no. $3,695-702$.

[20] H. Tuenter, A symmetry of power sum polynomials and Bernoulli numbers, Amer. Math. Monthly 108 (2001), no. 3, 258-261.

[21] S. Yang, An identity of symmetry for the Bernoulli polynomials, Discrete Math. 308 (2008), no. 4, 550-554.

Department of Mathematics

SOGANG University

SEOUl 121-742, Korea

E-mail address: dskim@sogang.ac.kr 